\title{
Exclude Clinical Observation Data From Statistics Indicator
}

National Cancer Institute

\section{Source}

National Cancer Institute. Exclude Clinical Observation Data From Statistics Indicator. NCI

Thesaurus. Code C119802.

Specifies whether the clinical observation result values should be excluded from the statistical analysis. 